Supplement of Saf. Nucl. Waste Disposal, 1, 279-280, 2021

https://doi.org/10.5194/sand-1-279-2021-supplement

(c) Author(s) 2021. CC BY 4.0 License.

(c) (1)

Supplement of

\title{
The use of muon radiography in safeguarding geological repositories
}

Lee Thompson et al.

Correspondence to: Lee Thompson (1.thompson@sheffield.ac.uk)

The copyright of individual parts of the supplement might differ from the article licence. 


\section{Crafe}

\section{The use of Muon Radiography in}

Safeguarding Geological Repositories

Lee Thompson, University of Sheffield

on behalf of

Katharina Aymanns, Irmgard Niemeyer, Christiane Vieh, Michael Weekes

12th November 2021

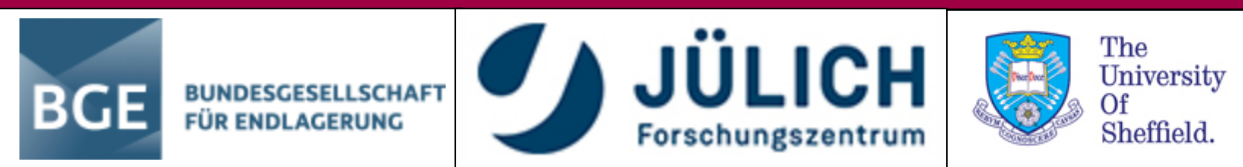




\section{Muons and Muon Tomography}

\section{What is muon tomography?}

- Muons are omnipresent fundamental particles that we are constantly bathed in

- They are created in the upper atmosphere

- From an imaging perspective they are both plentiful ( 1 per $\mathrm{cm}^{2}$ per minute) and free

- Importantly they are highly penetrating and pass through many tens of metres of rock

- Permits non-invasive, nondestructive imaging
- Works in exactly the same way as medical x-ray imaging

- A beam of $x$-rays (muons) passes through the object of interest

- A "detector" (film or digital system) is placed on the other side of the object of interest

- Density differences in the object are evident in the "image"

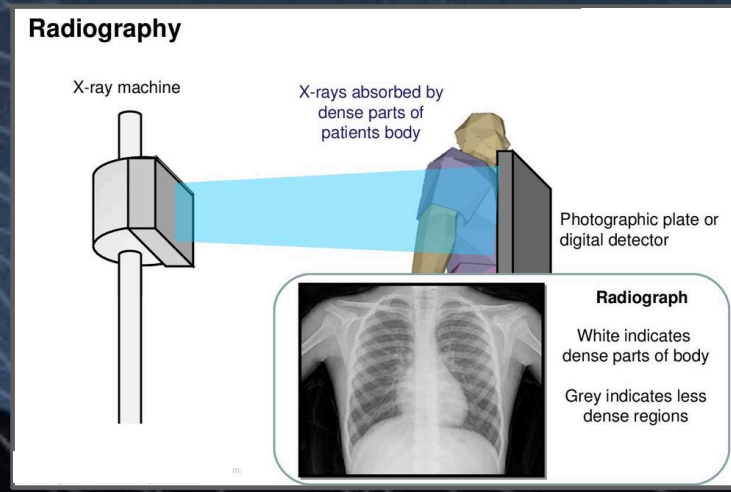

- However there are differences: muons are free and more penetrating than $X$-rays 


\section{Muon Tomography Track Record}

- This is not a new technique, it was first used to measure tunnel overburdens in 1955 and has been famously used to image pyramids and the magma chambers of volcanoes

- Currently undergoing a huge renaissance with many applications
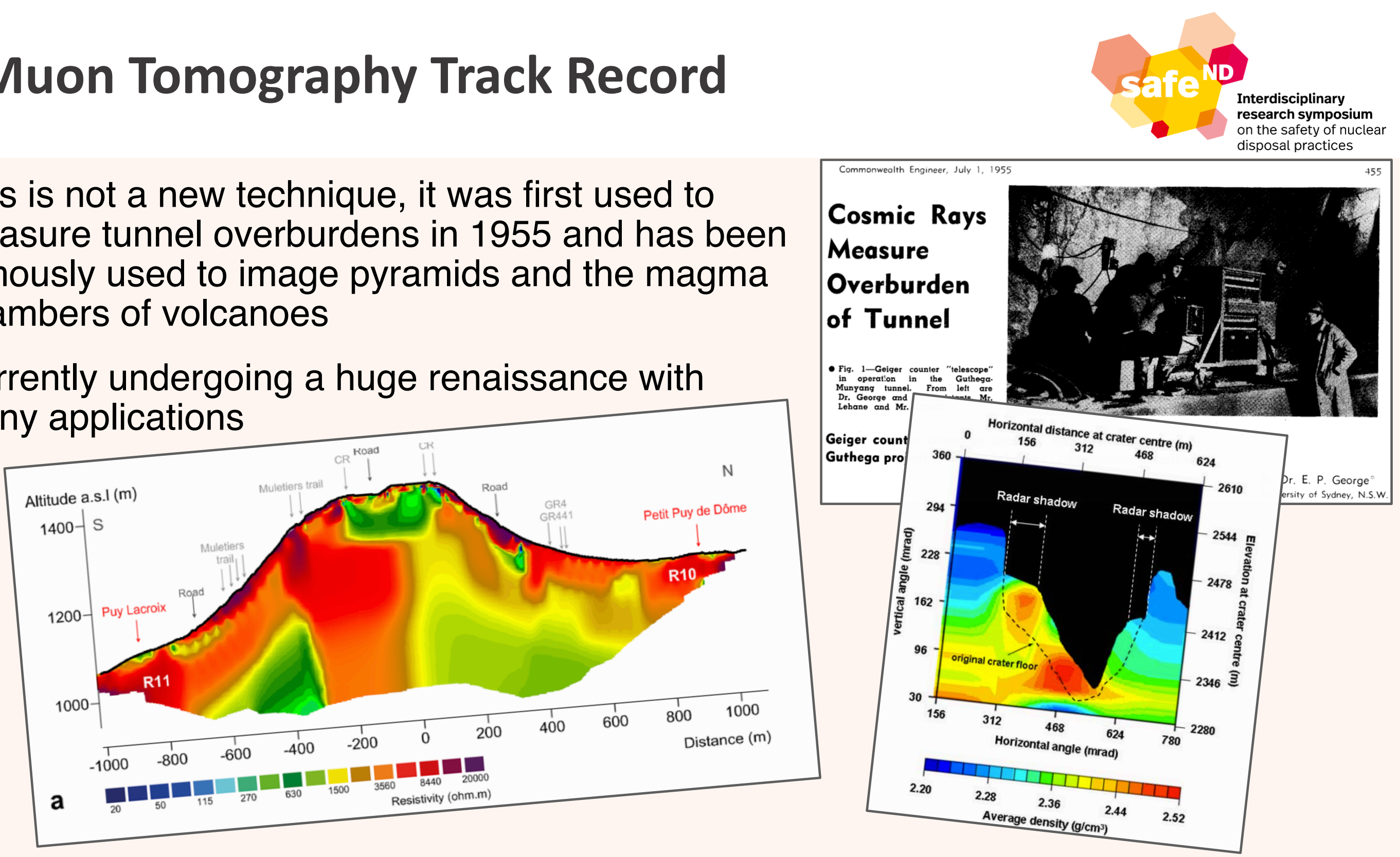

${ }_{20} \square_{50} \square_{115} \square_{270} \square_{630}{ }_{1500}{ }_{3560} \square_{8440} \square_{20000}$ 


\section{Applications of Muon Tomography}

- Eivil infrastructure:

- Tunnels unknown voids,

- Viaducts

- Bridges etc.

- Blast furnace imaging

- Glaciers - ice thickness measurements

- Volcanoes - imagma chanbers

- Mining - ore body imaging

- Stored $\mathrm{CO}_{2}$ (carbon capture and storage)

- Pyramids / Archaeology

- Imaging during nuclear waste storage and/or disposal

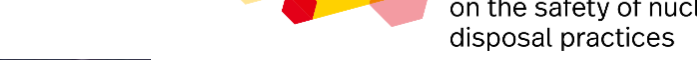




\section{Muon Tomography and Geological Repositories (GRs)}

- Civil infrastructure imaging and imaging of ore bodies in mines with muons is already underway

- For example: in the UK the technique is being used to search for hidden shafts in railway tunnels

- Elsewhere in the world nickel and uranium ore bodies are being located without the need for drilling boreholes
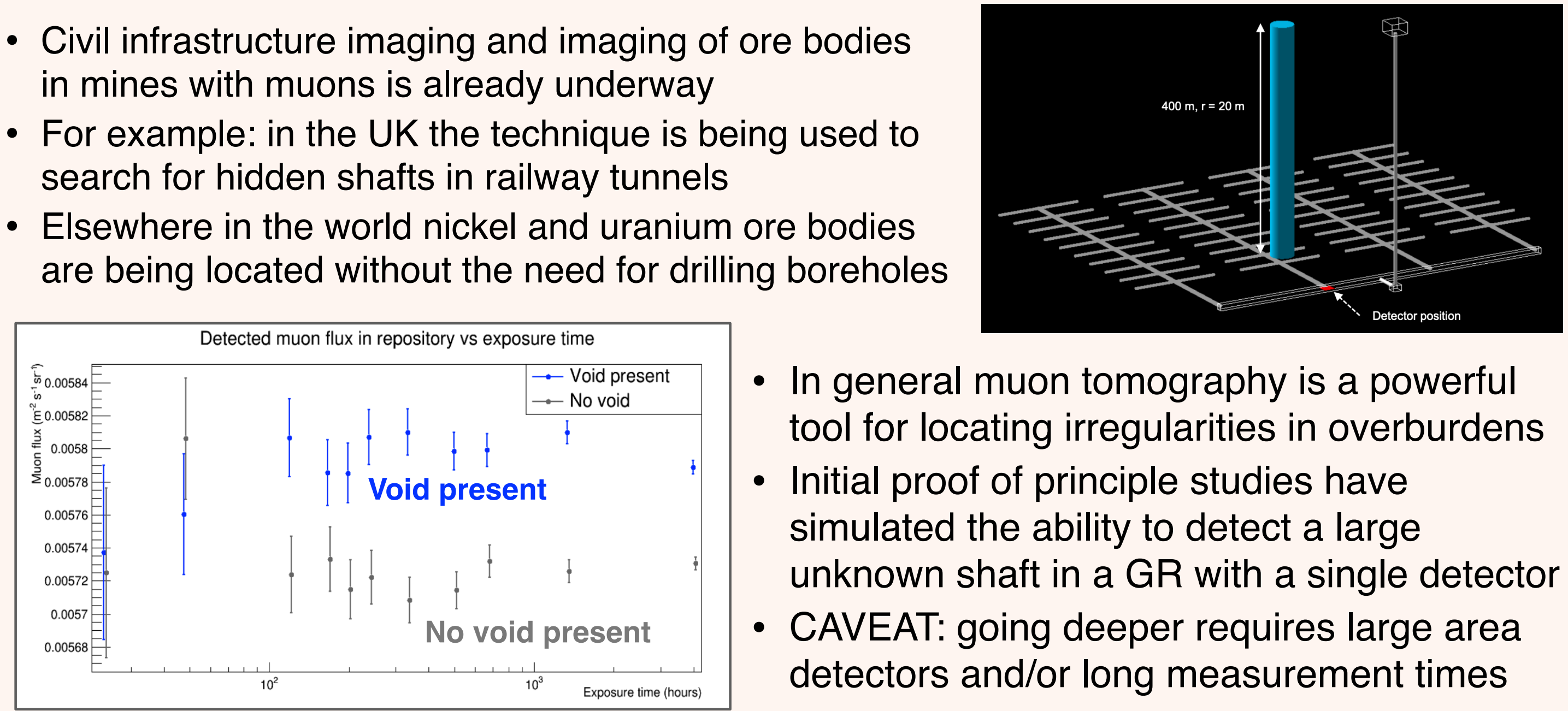

- In general muon tomography is a powerful tool for locating irregularities in overburdens

- Initial proof of principle studies have simulated the ability to detect a large unknown shaft in a GR with a single detector

- CAVEAT: going deeper requires large area detectors and/or long measurement times 


\section{Muon Tomography and Geological Repositories (GRs)}

- Other studies have looked at the detectability of an unknown feature in the GR as a function of the solid angle that the feature presents at the detector

- Note: multiple detectors plus imaging techniques such as SART and/or use of machine learning methods should considerably reduce the time needed to detect a feature

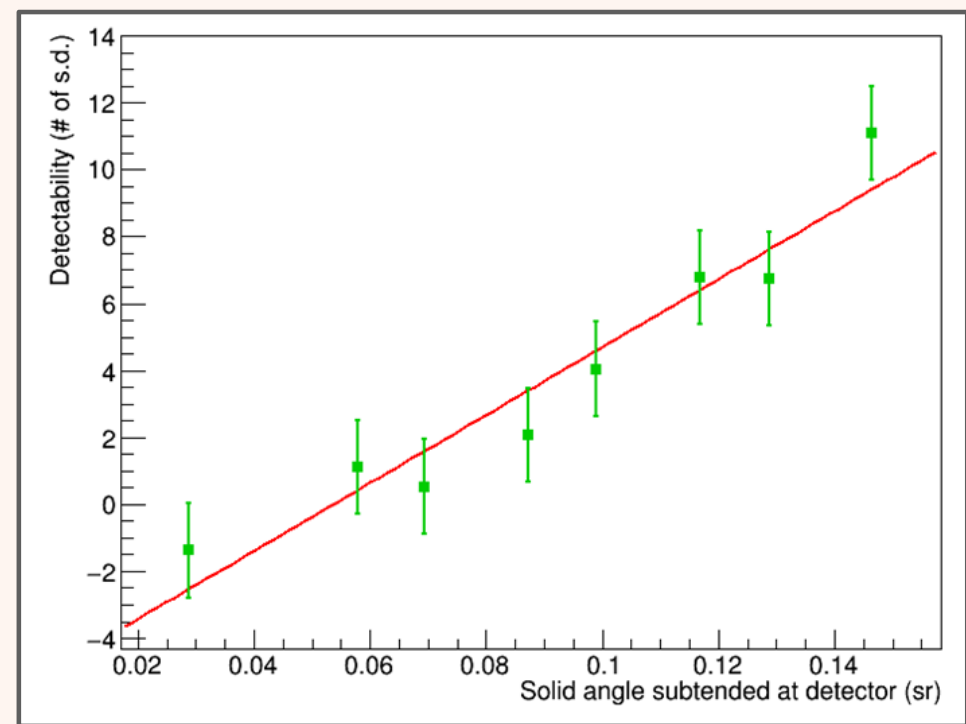

Possible application to GRs:

- design information verification

- continuous geological overburden monitoring for overburden change detection

- understanding the condition of the host geology

- searching for undocumented voiding

- checks of backfill integrity in the vaults

- tunnel lining system checks/monitoring

- sensitivity to water ingress and movement in the overburden

- long-term monitoring of the GR post-closure REMINDER: muon tomography is non-invasive and nondestructive

COMMENT: data fusion from seismic and muon radiography studies will be beneficial in some of these applications (resolves all material properties) 


\section{Muon Tomography - Other Methods}

\section{Muon Radiography ("Muography”)}

- Works in exactly the same way as medical $x$-ray imaging

- A beam of $\mathrm{x}$-rays (muons) passes through the object of interest

- A "detector" (film or digital system) is placed on the other side of the object of interest

- Density differences in the object are evident in the "image"

- However there are differences: muons are free and more penetrating than $\mathrm{X}$ rays

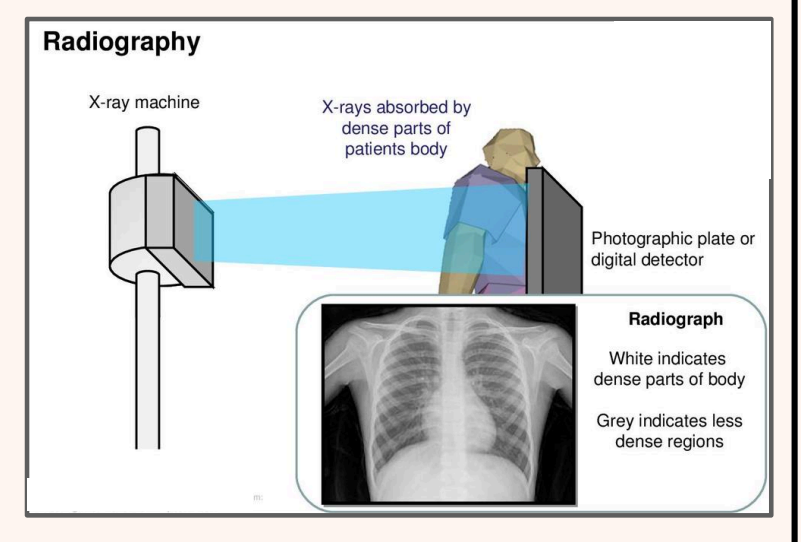

\section{Muon scattering tomography (“MST")}

- By placing muon detectors both above and below (or either side) of the object of interest additional information (about the nuclear composition) of the object being imaged can be determined

- Larger scattering angles correspond to materials with high atomic number $Z$

$\left.\sigma=\frac{13.6 \mathrm{MeV}}{p c \beta} z \sqrt{X\left(X_{0}\right)} 1+0.038 \ln \left(X / X_{0}\right)\right)$
$X_{0}=\frac{A \cdot 716.4 \mathrm{~g} / \mathrm{cm}^{2}}{Z(Z)+1) \ln (287 / \sqrt{Z})}$

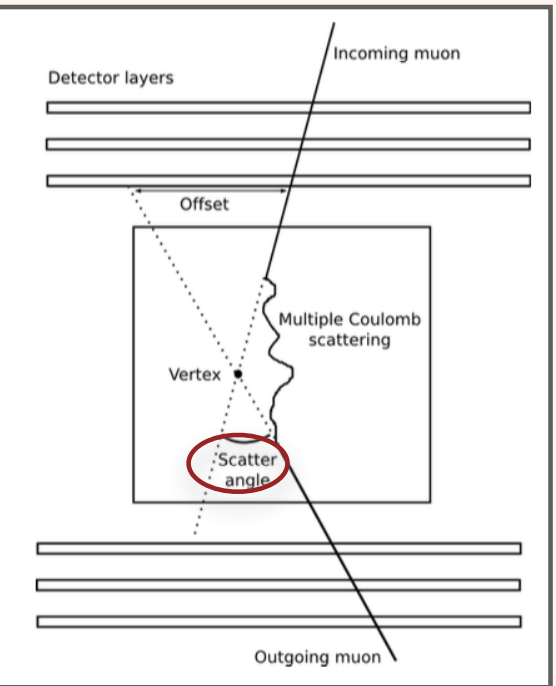




\section{Bubble ID in Heterogenous Waste Drums}

- Gas bubbles can form within the matrix of a waste drum and are a concern. Using muon scattering tomography bubbles can be identified and their volume accurately determined

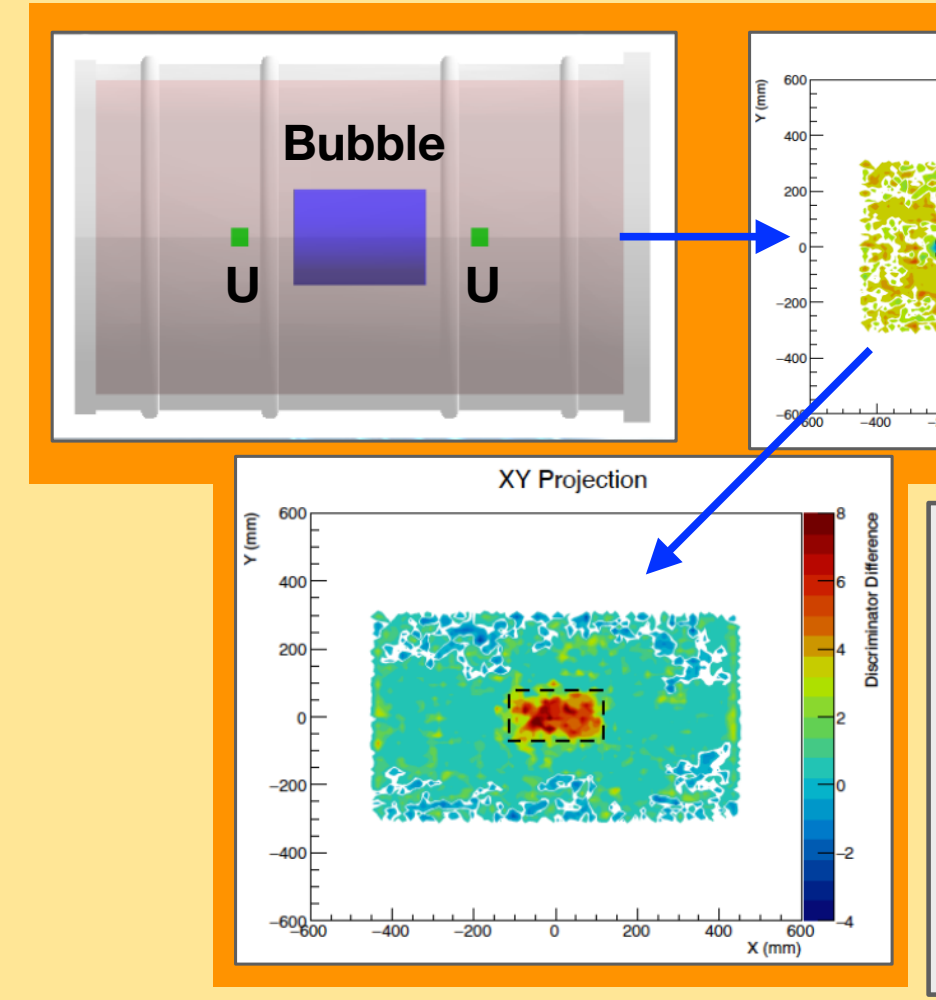

$\mathrm{XY}$ Projection

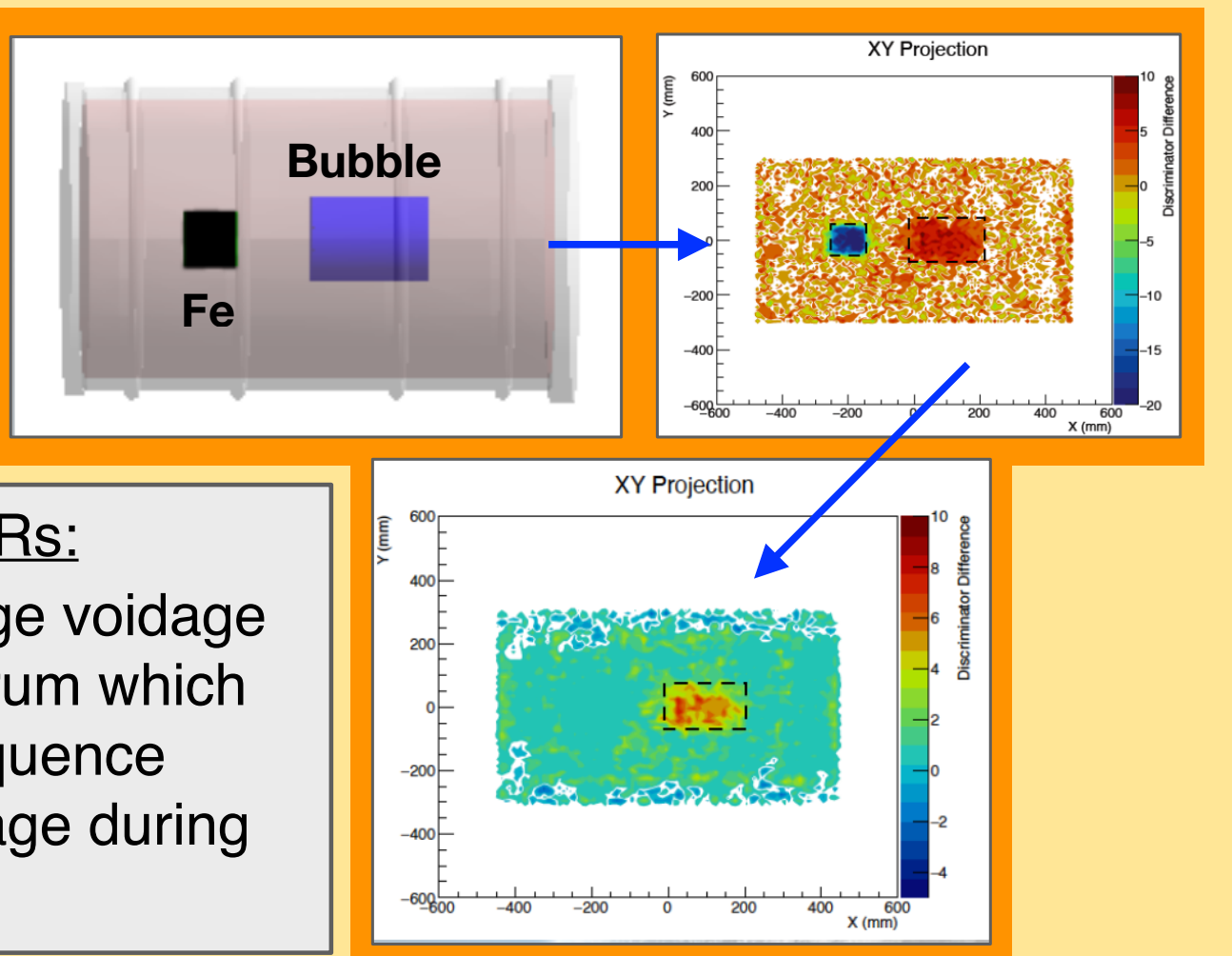

Possible application to GRs:

- monitoring of in-package voidage within nuclear waste drum which may result as a consequence settlement in the package during transportation 


\section{Material ID in Heterogenous Waste Drums}

A method has been developed to perform material identification using machine learning techniques STEP 1: identification of material boundaries in the waste drum which has a concrete matrix

STEP 2: uses machine learning MVA algorithms to assign a probability for each identified object being a particular material. See https://arxiv.org/abs/ 2012.01554

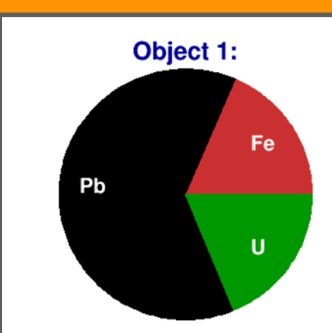

Ob 1: vol $785.0 \mathrm{~cm}^{3}$

Fe: $0.189+/-0.017$

$\mathrm{Pb}: 0.637+/-0.048$

U: $0.191+/-0.019$

Decision: U

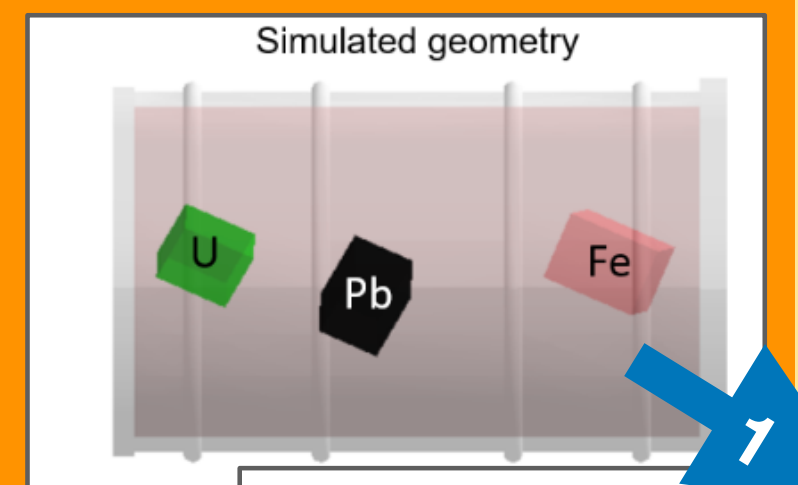

Identified clusters, $\mathrm{z}=0.0 \mathrm{~mm}$

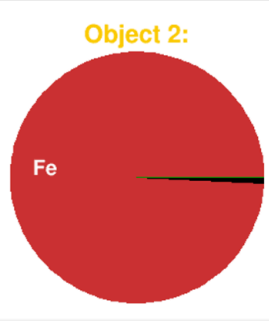

Fe: $0.993+/-0.049$,

$\mathrm{Pb}: 0.007+/-0.003$,

U: $0.000+/-0.000$ Decision: Fe

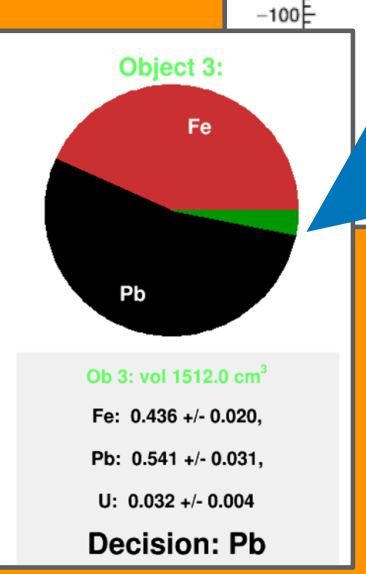

This project has received funding from the European Union's Horizon 2020 research and innovation programme under grant agreement number 755371.
Possible applications to GRs:

- safeguarding any outgoing potentially-empty package (e.g. MST would be able to confirm, quickly, any presence of high-Z material in the outgoing package that shouldn't be there)

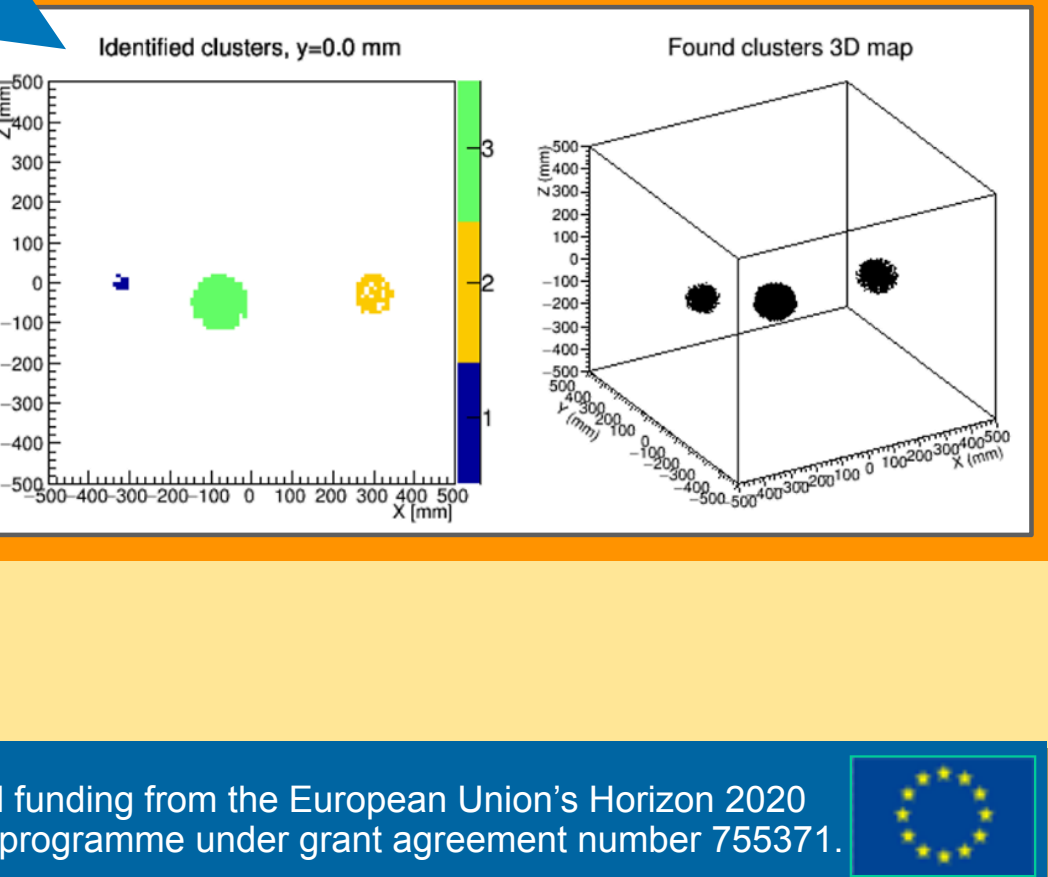




\section{Safeguards Applications}

Looking at the potential for muon scattering tomography to identify possible changes to a CASTOR drum

Possible applications to GRs:

- confirming that a full complement of in-package components is present (no unauthorised diversion of materials)

- confirmation that out-going packages are truly empty
Diversion Scenarios considered:

1.Empty basket

2. Half-loaded basket (Unloaded side fuel assemblies) 3. Half-loaded basket (Unloaded centre fuel assemblies) 4. $\mathrm{Pb}$ pellets basket $\left(\mathrm{UO}_{2}\right.$ pellets replaced by $\mathrm{Pb}$ pellets $)$
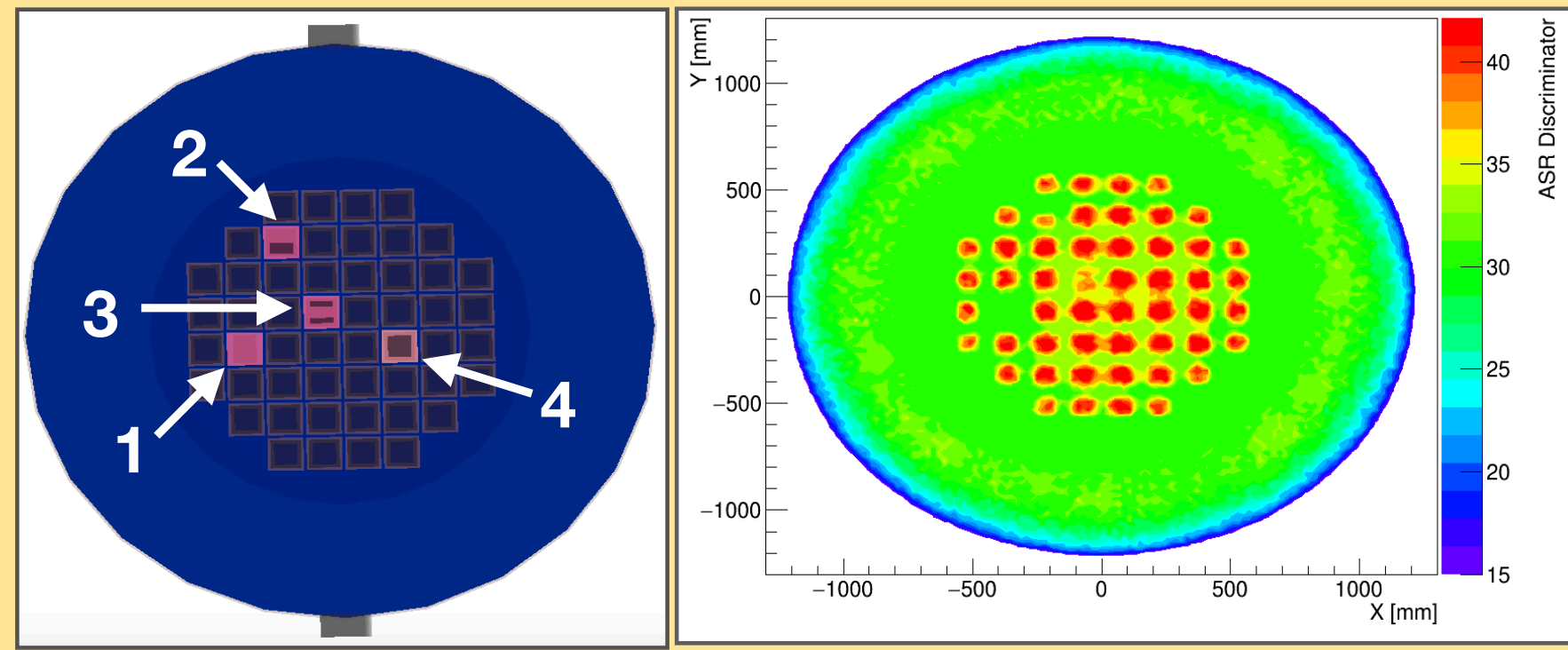

This project has received funding from the European Union's Horizon 2020 research and innovation programme under grant agreement number 755371 . 


\section{Conclusions}

- Muon tomography is a powerful tool that exploits naturally occurring radiation to form images of objects in a non-invasive and non-destructive way

- It has been famously used to search for hidden chambers in pyramids and to image the magma chambers in volcanoes

- The technique is currently applied globally to a huge range of applications including imaging of civil infrastructure, mines, núclear safeguards and material control, homeland security

- Within the management of nuclear waste there are a number of areas where muon radiography is a promising technology to address specific problems such as geological repository design information verification, integrity assurance and longterm monitoring

- Similarly, muon scattering tomography offers the possibility to identify issues such as material diversion, package voiding and material identification. 